\title{
Dimensions de la vulnérabilité liée au paludisme dans deux zones de Madagascar : apports d'une approche mixte
}

Dimensions of malaria-related vulnerability in two areas of Madagascar:

contributions of a mixed approach

Andry Herisoa Andrianasolo, Emma Raboanary, Chiarella Mattern, Thomas Kesteman, Dolorès Pourette et Christophe Rogier

\section{(2) OpenEdition Journals}

Édition électronique

URL : https://journals.openedition.org/popvuln/1052

DOI : 10.4000/popvuln. 1052

ISSN : 2650-7684

Éditeur

LIR3S - Laboratoire Interdisciplinaire de Recherche "Sociétés Sensibilités Soin" (UMR 7366 CNRS-uB)

Édition imprimée

Date de publication : 1 mars 2019

Pagination : $129-156$

ISBN : 978-2-918173-26-7

ISSN : 2269-0182

\section{Référence électronique}

Andry Herisoa Andrianasolo, Emma Raboanary, Chiarella Mattern, Thomas Kesteman, Dolorès Pourette et Christophe Rogier, « Dimensions de la vulnérabilité liée au paludisme dans deux zones de Madagascar : apports d'une approche mixte », Populations vulnérables [En ligne], 5 | 2019, mis en ligne le 26 mai 2020, consulté le 26 février 2022. URL : http://journals.openedition.org/popvuln/1052 ; DOI : https://doi.org/10.4000/popvuln.1052

Ce document a été généré automatiquement le 26 février 2022.

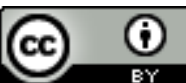

Les contenus de la revue Populations vulnérables sont mis à disposition selon les termes de la Licence Creative Commons Attribution 4.0 International 


\title{
Dimensions de la vulnérabilité liée au paludisme dans deux zones de Madagascar : apports d'une approche mixte
}

\author{
Dimensions of malaria-related vulnerability in two areas of Madagascar: \\ contributions of a mixed approach
}

Andry Herisoa Andrianasolo, Emma Raboanary, Chiarella Mattern, Thomas Kesteman, Dolorès Pourette et Christophe Rogier

Étudefinancée par le dispositif «Initiative $5 \%$ - Fonds Mondial » du ministère français des Affaires étrangères, l'Institut Pasteur de Madagascar, l'Institut pour la recherche et le d éveloppement (IRD-DPF, Programme ARTS).

\section{Introduction}

1 Au cours de la dernière décennie, le poids du paludisme a reculé à Madagascarmais la situation reste alarmante. La morbidité est restéeautour de $5 \%$ à $6,5 \%$ entre 2011 et 2013 (ministère de la Santé publique de Madagascar et Roll back malaria, 2016) et une recrudescence inattendue du paludisme a été observée dans le Sud-Ouest de la Grande Île durant le premier semestre de 2012. Le nombre de cas signalés a été multiplié par 2,5 à 10 lors de cette épidémie qui a touché toutes les catégories d'âge de la population. Cette zone qui était endémique à transmission pérenne, épargnée autrefois par les épidémies, y était devenuesensible (ministère de la Santé publique de Madagascar, 2012).

2 Le paludisme est une maladie infectieuse due aux parasites du genre Plasmodium. À Madagascar, $90 \%$ des cas de paludisme sont causés par l'espèce $P$. falciparum (Aubry et Gaüzère, 2015). Le parasite du paludisme se transmet, la nuit, lors de la piqûre par un 
moustique femelle du genre Anopheles. P. falciparum peut entraîner des complications puis la mort en cas de prise en charge tardive du patient.

\section{Objectifs et problématique}

Dans le contexte d'une volontéaffichée du gouvernement malgache et de ses partenaires financiers et techniques d'atteindre les objectifs d'un développement durable en matière de paludisme (ministère de la Santé publique de Madagascar, 2012 ; OMS, 2015), et dans une démarche de mise en œuvre d'un système de couverture médicale universelle pour la population malgache, cette analyse a pour principal objectif d'apporter des éléments de réflexion sur l'adaptabilité des mesures de lutte à la réalité du terrain, en identifiant les déterminants de la vulnérabilité au paludisme de cette population.

Il s'avère important de comprendre pourquoi le paludisme reste un problème de santé publique à Madagascar malgré les efforts consentis pour la lutte.

5 Pour répondre à cette question, le contexte de prévalence des infections plasmodiales et l'accès aux soins et à la santé en cas de fièvre présumée palustre ont été étudiés. L'analyse s'est concentrée, dans deux districts, sur des déterminants multidimensionnels (Becerra, 2012) de la vulnérabilité au paludisme :

- à caractère géoclimatique et épidémiologique,

- relatifs aux interventions de lutte contre le paludisme, ou

- socio-économiques et comportementaux.

\section{Matériels et méthodes}

Les données ont été collectées en 2014.

\section{1) Zones d'étude}

7 Deux districts ont constitué les zones d'étude. Les districts d'Ankazobe et de Brickaville, distants de $300 \mathrm{~km}$ l'un de l'autre, ont été choisis pour leurs différences épidémiologiques et bioclimatiques, pour leur diversité socio-culturelle et pour la particularité des actions de lutte contre le paludisme réalisées dans chacun d'eux. Brickaville est localisé sur la côte est de la Grande Île, à $220 \mathrm{~km}$ de la capitale. Ankazobe est situé sur les Hautes Terres, à $70 \mathrm{~km}$ au nord de la capitale Antananarivo, à 1253 mètres d'altitude. Deux communes par district ont été choisies selon leur accessibilité par rapport aux formations sanitaires. 
Figure 1. Carte de Madagascar et localisation des districts d'Ankazobe (en rouge) et de Brickaville (en bleu)

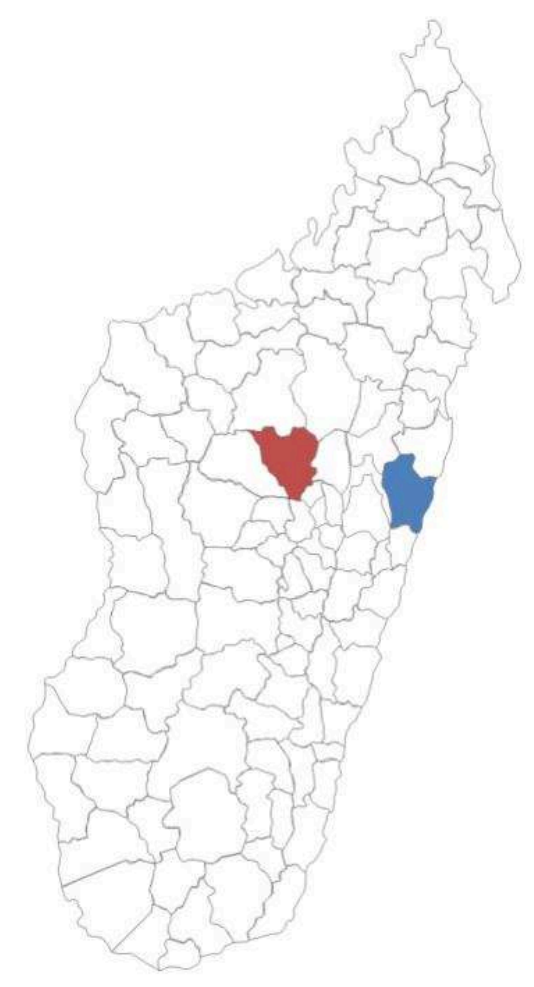

\section{2) Méthode mixte}

8 Une approche mixte a été utilisée. La pertinence de l'utilisation de méthodes qualitative et quantitative combinées est soutenue dans des études d'évaluation de programmes, notamment en santé publique (Pluye et Hong, 2014 ; Pluye, 2012; Ridde, 2013). Les résultats préliminaires de l'analyse des données qualitatives ont permis d'ajuster le questionnaire de l'enquête quantitative (AIMS, 2010 ; Hunt et Lavoie, 2011).

\section{3) Production des données qualitatives : entretiens individuels}

Au total, 62 entretiens semi-directifs ont été conduits en face à face avec des acteurs de la lutte contre le paludisme (Angers, 1996; Houéto, D’Hoore et Deccache, 2008). Il s'agissait d'habitants (hommes, femmes, femmes enceintes, malades, non malades), de soignants de centres de santé de base ${ }^{2}$, d'agents communautaires (AC) ${ }^{3}$, de guérisseurs traditionnels, d'un maire, de chefs de fokontany ${ }^{4}$, de chefs traditionnels, de personnel d'école primaire publique, de cadres des services de santé de district (SSD), de responsables d'organisations non gouvernementales partenaires et de colporteurs de médicaments. Des discussions informelles et des informations par observation ont complété ces entretiens. L'identification des participants suivait le principe de «la boule de neige». La collecte des données a été poursuivie jusqu'à «saturation des informations". 


\section{4) Collecte et analyse des données quantitatives}

10 L'approche quantitative comprenait une enquête à dimension sociodémographique, épidémiologique et économique dans la population. En considérant les ménages comme unité statistique, et afin de pouvoir estimer un taux de $50 \%$ avec un intervalle de confiance bilatéral à $95 \%$ et une précision de $5 \%$, il était nécessaire de recruter au minimum 400 ménages par district. En estimant que la taille moyenne des ménages était de 5 individus, l'échantillon attendu était de 2000 personnes par district. Les ménagesont été sélectionnés par cheminement aléatoire au sein des villages.

11 Ainsi, les échantillons d'Ankazobe et de Brickaville comptaient respectivement 2101 individus et 1942 individus, soit au total, 4043 individus, issus de 803 ménages. Les individus inclus représentaient en moyenne $95 \%$ des individus sollicités.

L'effet " grappe » dû à la non-indépendance des observations effectuées dans un même fokontany (i.e. quartier) a été contrôlé dans les analyses en utilisant les fonctions "svy » du logiciel Stata adaptées au plan d'échantillonnage, i.e. stratification par district et agrégation par fokontany. Les fonctions «svy» ont permis de tenir compte du plan d'échantillonnage dans l'estimation des variances pour le calcul des intervalles de confiance et les tests de significativité en régression logistique ou linéaire, bi- ou multivariée.

\section{Vulnérabilité liée aux conditions bioclimatiques}

Quatre faciès (équatorial, sahélien, tropical, de montagne)épidémiologiques sont décrits selon le contexte bioclimatique des zones à Madagascar (Aubry et Gaüzère, 2015 ; INSTAT, 2010). Les conditions bioclimatiques chaudes et humides de la côte est (zone de Brickaville) favorisent le développement des larves de moustiques vecteurs des plasmodiums, et entraînent une exposition pérenne de la population au paludisme. Son incidence y est généralement élevée. Brickaville présente un faciès de paludisme de type équatorial. Le faciès équatorial se caractérise par une forte transmission des plasmodiums tout le long de l'année. À Ankazobe, le climat sec et tempéré ne convient pas à la longue survie des moustiques. L'incidence du paludisme y est relativement faible, et la transmission des plasmodiums est observée entre avril et juin. Ankazobe présente un faciès de montagne avec un paludisme instable et présentant un profil épidémique lié à sa transmission saisonnière. La vulnérabilité liée au paludisme est donc plus grande à Brickaville qu'à Ankazobe.

Le graphique suivant montre la différence de prévalence de la fièvre et de l'infection plasmodiale entre les deux districts pendant les trois mois précédant l'enquête. Le nombre d'individus étant décédés du paludisme donne un ordre d'idée de la différence du taux de mortalité attribuée au paludisme entre les deux zones. 
Figure 2. Fréquence du paludisme, de la fièvre et des décès attribués au paludisme

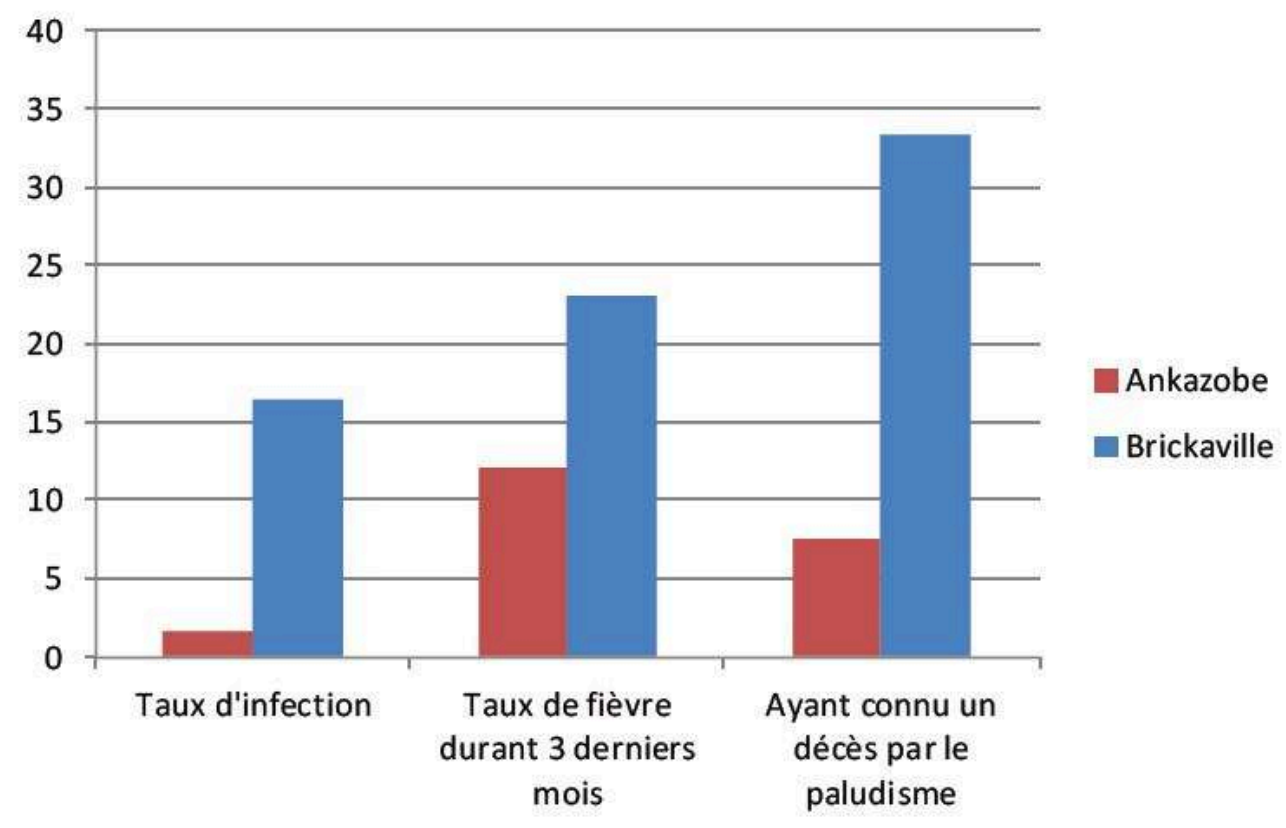

\section{Vulnérabilité liée aux activités de lutte contre le paludisme et aux pratiques des soignants}

À Madagascar,la prévention, le diagnostic et le traitement du paludisme sont pris en charge par le programme national de lutte contre le paludisme (PNLP) et sont donc supposés gratuits pour la population. Les opérations de lutte consistent notamment à :

- diagnostiquer le paludisme par un test de diagnostic rapide (TDR) ;

- traiter les malades atteints de paludisme par Artemisinin-based combination therapy (ACT);

- distribuer des moustiquaires imprégnées d'insecticide de longue durée (MILD) à la population ;

- administrer un traitement préventif intermittent à base de sulfadoxine-pyriméthamine, aux femmes enceintes ;

- réaliser une campagne d'aspersion intra-domiciliaire d'insecticide dans les ménages des zones à faible transmission ;

- sensibiliser et éduquer la population à la lutte contre le paludisme par des séances d'Information-éducation-communication (IEC).

Le graphique suivant (Figure 3) montre le niveau de réalisation des actions de lutte

à Ankazobe et à Brickaville. Ces données concernent les individus ayant eu de la fièvre et ayant consulté un personnel médical, durant les trois mois précédant l'enquête.

L'utilisation de TDR étaitfaible à Ankazobe. Un peu plus de la moitié des individus venus consulter pour une fièvre ont été diagnostiqués par TDR à Brickaville. Le taux de prise d'ACT était relativement élevé mais n'était pas conforme à ce qui était attendu. Brickaville se distinguait aussi par la pratique significativement plus fréquente de l'injection de quinine en cas de fièvre. L'injection de quinine n'est justifiée que dans les formes compliquées ou graves de paludisme. Elle devrait être exceptionnelle. À 
Brickaville, il semble donc exister un excès important d'utilisation de ce médicament par injection intramusculaire.

18 L'approche qualitative a permis d'éclairer les causes de ces anomalies apparentes dans la prise en charge des fièvres et du paludisme.

\section{1) Le diagnostic du paludisme}

En l'absence de méthode diagnostique fiable (microscopie et TDR, notamment), l'identification des cas de paludisme reposait sur la présomption de paludisme en présence de fièvre, de quelques autres signes cliniques peu spécifiques et en l'absence de signes évoquant une autre pathologie. Le manque de spécificité de cette approche diagnostique a pu entrainer un traitement abusif des cas de fièvre par des antipaludiques (favorisant la sélection et l'extension de la résistance de P. falciparum aux antipaludiques) et une surestimation de l'incidence du paludisme (Rakotomalala et al., 2015).

Figure 3. Fréquences des actions de prise en charge du paludisme chez les patients ayant eu de la fièvre et ayant consulté un personnel médical $\left(N_{A}=85\right.$ et $\left.N_{B}=264\right)$

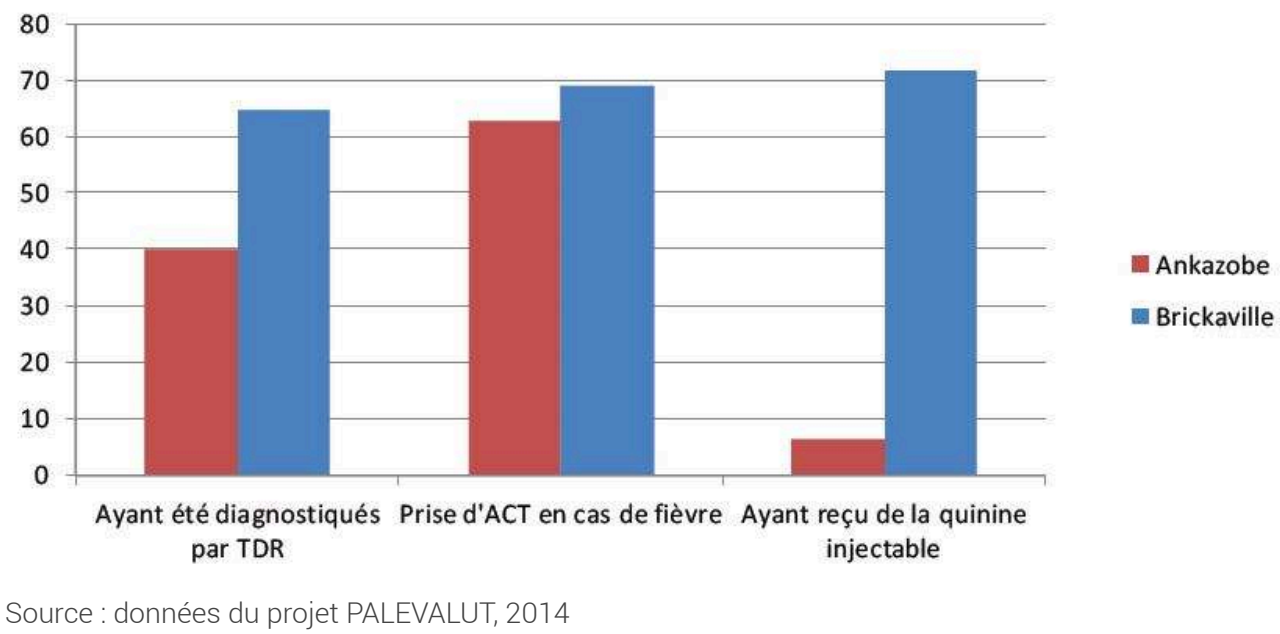

Dans les deux zones d'étude, la rupture de stocks de TDR a été évoquée fréquemment ${ }^{5}$, par les habitants, les soignants et les responsables dans les SSD. Cela a pu avoir un impactnégatif sur les pratiques des soignants mais aussi sur l'estimation du nombre de cas de paludisme.

21 L'absence d'utilisation de TDR entraînait une confusion entre fièvres dues au paludisme et celles dues à d'autres pathologies. Par conséquent, les traitements prescrits n'étaient pas toujours appropriés. Le verbatim suivant rapporte le cas d'une fièvre pesteuse prise pour une fièvre palustre, à cause de l'absence de TDR.

Une fois, je me suis trompé totalement et cela a coûté la vie d'une personne. [...] La grosse fièvre m'a fait penser directement au paludisme compliqué [...]. De plus, je n'avais pas aperçu de bubon sous les aisselles pour penser à la peste bubonique [...]. (médecin)

Le médecin injecta de la quinine ${ }^{6}$ au malade mais ce dernier mourût le lendemain. Un test biologique post-mortem réalisé par le SSD a permis d'identifier une infection par Yersinia pestis, l'agent de la peste. 

précis, sur un mode probabiliste, presque « au hasard».

Je me demande à chaque fois si c'est du paludisme ou non [...]. Je n'ose pas prescrire un traitement de paludisme uniquement car j'ai peur qu'il s'agisse d'une autre maladie. Je donne un traitement antipaludique et j'ajoute d'autres médicaments. Les malades en sont guéris, mais on ne sait plus si c'était du paludisme et donc traité, ou si c'était l'effet des autres médicaments... C'est comme ça que je vis les choses! [Rire]. (médecin)

Le manque de TDR faussait les statistiques sur le nombre de cas de paludisme. En fait, le nombre de cas de paludisme déclaré au SSD devait être conforme au nombre de TDR utilisés.Or, avec la rupture de stocks de TDR, des cas de paludisme présumés sur la base des signes cliniques ont tout de même été traités par des antipaludiques par les soignants. Il s'agit d'une pratique appropriée en l'absence de TDR, pour sauvegarder les chances de guérison des patients. Mais, selon les soignants, ces déclarations de cas de paludisme en nombre supérieur à celui des TDR utilisés, étaient interprétées dans les SSD comme une surestimation du nombre de cas par les soignants des centres de santé de base, dans le but d'obtenir plus de TDR. Cependant, la non-déclaration des cas de paludisme non diagnostiqués par TDR limitait aussi la justification de l'approvisionnement en ACT, augmentant ainsi les risques de rupture de stocks de médicaments.

Depuis que le diagnostic repose sur l'utilisation de TDR plutôt que sur la présomption de paludisme en cas fièvre, le nombre de cas déclarés de paludisme a baissé (Direction de la Veille sanitaire et de la surveillance épidémiologique, 2012 ; Rakotomalala et al., 2015). Cette baisse apparente de l'incidence du paludisme est possiblement due, en partie, à la non-déclaration des cas non diagnostiqués par TDR, faute d'intrants. Un cercle vicieux de rupture de stocks de TDR provoquant une sous-déclaration du paludisme et limitant finalement les possibilités de commande des TDR a pu s'installer. Des ruptures de stocks de médicaments ont pu aussi être la conséquence de ce défaut.

\section{2) Le traitement du paludisme}

L'antipaludique ACT, le traitement de référence, est présenté sous trois formats suivant l'âge des patients: ACT Nourrisson, ACT Enfant et ACT Adulte. Les ACT doivent être prescrits à la suite d'un TDR positif (TDR+) pour le paludisme.

Le principal problème dans l'utilisation des ACT était lié aux ruptures de stocks de ces médicaments. Pour faire face à la situation, des soignants ont «bricolé ».Le manque d'ACT Adulte (le format le plus souvent en rupture de stocks), a conduit des soignants à doubler la dose d'ACT Enfant pour traiter des adultes, et inversement à fractionner une formulation pour adulte afin de traiter des enfants. Cette pratique expose à des erreurs de dosage potentiellement dangereuses pour le patient.

Effectivement, les ACT Adulte manquent contrairement aux ACT Nourrisson et ACT Enfant. Nous avons donné l'ordre aux médecins des centres de santé de base de prescrire des ACT Enfant aux adultes, en doublant la dose. Scientifiquement parlant, tenant compte des principes actifs, ... nous ne savons pas si c'est correct ou non... Pareillement, nous avons dicté de prescrire des ACT Enfant aux nourrissons, en réduisant la dose à moitié. [...] Cela ne fausse pas la comptabilité du nombre d'ACT prescrits [par rapport au nombre de TDR+ réalisés], car dès le départ, la quantité d'ACT Enfant commandée est doublée pour permettre les prescriptions pour les adultes.(cadre de SSD) 
Lorsque les ACT n'étaient pas disponibles dans les centres de santé de base, il était demandé aux patients et à leur famille d'en acheter sur le marché, suivi du « bricolage » de la posologie.

C'est l'approvisionnement en ACT qui pose le plus problème. Quand les ACT Adulte manquent, les patients sont priés d'acheter des ACT Enfant dans les dépôts de médicaments. (cadre d'un SSD)

Cependant, demander aux malades d'acheter des ACT sur le marché ne garantissait pas l'acquisition des médicaments par ces derniers, la prise de manière correcte d'au moins la première dose, la parfaite compréhension de la posologie du traitement et la qualité des médicaments (respect des conditions de conservation et de la date de péremption par exemple). Les médecins ont affirmé qu'il leur était impossible de suivre les traitements lorsque les médicaments devaient être acquis en dehors des centres de santé.

Chez les adultes, en l'absence d'ACT, le paludisme non compliqué était parfois traité par de la chloroquine (médicament dont l'utilisation contre le paludisme est proscrite à cause de la résistance de $P$. falciparum) associée à de la tétracycline ou de la doxycycline. La posologie appliquée était la suivante :

Il faut partager en deux le dosage de chloroquine de $10 \mathrm{mg} / \mathrm{kg} / \mathrm{jour}$... à prendre en deux prises (par jour), pendant trois jours au minimum à cinq jours, accompagné de tétracycline. Il faut $u$

tiliser de la tétracycline $250 \mathrm{mg}$. Il faut prendre 2 comprimés le matin, 2 comprimés à midi, et 2 comprimés le soir... c'est-à-dire $500 \mathrm{mg}$ trois fois par jour pendant 5 jours.(médecin)

Cette posologie combinant la chloroquine et une cycline dont la vitesse d'action contre les plasmodiums est insuffisante ne permet pas de traiter correctement le paludisme et expose le patient à un risque évolution vers un paludisme grave [C. Rogier, communication personnelle]. Elle n'a d' jamais été recommandée par l'OMS ou le PNLP.

L'approvisionnement régulier d'ACT Enfant et Nourrisson, contrairement aux ACT Adulte, semble être liée à une priorisation de la prise en charge des enfants, alors que les individus des autres classes d'âges étaient aussi concernés par le paludisme(Rogier et al., 2013). En outre, Madagascar a connu des difficultés d'approvisionnement en intrants suite à une suspension des financements du Fonds mondial liée à des mauvaises pratiques (corruption) de membres d'organismes nationaux en charge de l'approvisionnement (GF-OIG-13-052, 2014). Ces ruptures de stocks d'ACT pourraient avoir entraîné une surmortalité par paludisme qui était évitable.

\section{3) La prise en charge du paludisme grave}

D'après les consignes des SSD, conformes à celles du PNLP, les cas de paludisme grave ne devaient pas être traités au niveau des centres de santé de base, mais devaient être évacués dans un centre hospitalier.Cependant, le coût de l'évacuation sanitaire était souvent exorbitant pour les familles. Les soignants étaient suppliés par les familles de soigner sur place les malades. Ainsi, il arrivait souvent que des soignants prennent en charge des cas compliqués de façon délocalisée, avec administration par voie intraveineuse de doses de quinine, en dépit des recommandations. En outre, faute de capacité d'accueil dans la plupart des centres de santé de base, les malades sous perfusion étaient hébergés par des ménages aux alentours des formations sanitaires. 
Cette disposition rendait difficile le suivi du traitement des patients par les médecins. Ces derniers ont déclaré ne pas avoir le choix de faire autrement. Un soignant a aussi évoqué le coût des intrants à payer pour la perfusion (seringues, perfuseur, flacons de soluté glucosé à $5 \%$, ampoules injectables) en plus des médicaments autres que les ACT. Pour éviter de faire payer les flacons de soluté glucosé et le perfuseur aux familles, et de suivre pendant plusieurs jours les malades sous perfusion, un soignant a décidé d'administrer directement par voie intramusculaire les doses de quinine. D'après lui, cette pratique était bien connue des autres médecins.

Les spécialistes n'accepteront jamais, mais nous, les médecins sur le terrain, n'avons pas le choix. [...] Nous diluons la solution et nous l'injectons bien profondément en IM (intramusculaire). Le plus important pour nous, c'est la guérison des patients [...]. Il faut tout simplement donner de l'eau sucrée au malade, s'il arrive à boire, pour éviter l'hypoglycémie que peut entraîner la grande dose de quinine introduite d'un coup. (médecin)

Pourtant, l'injection intramusculaire de quinine est à l'origine d'abcès pouvant léser le nerf sciatique, et entraîner des paralysies définitives et des douleurs importantes (Bileckot et al., 1992). C'est pour cette raison que ce mode d'administration de la quinine est proscrit.

Le choix du PNLP d'imposer l'enregistrement des patients ayant un paludisme compliqué auprès des structures hospitalières est justifié sur le plan théorique mais se révèle inadapté sur le terrain, poussant les soignants à adopter des pratiques inappropriées et potentiellement dangereuses.

\section{4) La mise en œuvre des mesures préventives}

Les données géoclimatiques et épidémiologiques justifiaient la différence des stratégies de lutte menées à Brickaville et àAnkazobe. L'aspersion intra-domiciliaire d'insecticide réalisée à Ankazobe visait à réduire la densité et le temps de survie des moustiques vecteurs qui se reposent, après leurs repas sanguins nocturnes, à l'intérieur des maisons. Ce type d'action a surtout prouvé son intérêt dans les zones où les moustiques se reposent plus à l'intérieur qu'à l'extérieur des maisons (comme à Ankazobe), contrairement à leur comportement dans les zones où les conditions climatiques sont plus favorables à l'extérieur des maisons (comme à Brickaville) [C. Rogier, communication personnelle].

Quant aux MILD, la dernière campagne de distribution avant l'enquête s'était déroulée en 2011 et 2012. Le quota de moustiquaires des ménages était fixé à 1 MILD/3 personnes. Dans notre étude, les participants âgés de 15 ans et plus (4 041 individus) ont été interrogés sur le nombre de fois où ils ont dormi la nuit sous une moustiquaire durant les deux semaines précédant le passage des enquêteurs. Plus d'individus ont dormi « toutes les nuits »sous moustiquaires à Brickaville (96,5\%) qu'à Ankazobe (70 \% ; OR=12,8; IC $95 \%: 5,68-28,8 ; p<0,001$; Figure 4). Parmi les 587 individus qui n'avaient pas dormi régulièrement sous moustiquaires, $24,5 \%$ (IC $95 \%: 13,3-35,8$ ) ont évoqué l'insuffisance du nombre de moustiquaires disponibles. Les entretiens qualitatifs ont permis d'apprendre que même si le quota d'une MILD pour trois personnes était respecté lors de la distribution, il était inévitable que des individus dormissent hors moustiquaires. En réalité, certaines catégories de personnes ne pouvaient pas dormir ensemble pour des raisons coutumières ou de confort (par exemple, un frère et une 
sœur sont interdits par la coutume de dormir ensemble, à partir de l'adolescence ; un adulte nepeut pas partager le même lit que ses parents).

La proportion de femmes enceintes ayant pris des antipaludiques préventifs lors des consultations prénatales était faible $\left(28,4 \%\right.$ pour $\mathrm{N}_{\mathrm{A}}=134 ; 43,3 \%$ pour $\mathrm{N}_{\mathrm{B}}=68$; Figure 4$)$. La prise d'antipaludiques était deux fois moins fréquente à Ankazobe qu'à Brickaville (OR=0,5; IC $95 \%: 0,2-0,8 ; p=0,018)$, sans différence significative en fonction de l'âge de la femme et de l'âge de la grossesse.

Depuis la rupture de stocks de sulfadoxine-pyriméthamine en 2013 dans ces districts, il était demandé aux femmes enceintes d'en acheter sur le marché. Mais devoir acheter le médicament augmentait le coût de la consultation prénatale. Bien que théoriquement prise en charge par le PNLP et donc gratuite pour les gestantes, la consultation prénatale était payante pour $64,4 \%$ des femmes enceintes d'Ankazobe et $33,8 \%$ des femmes enceintes de Brickaville. Ensuite, demander aux gestantes d'acheter le traitement préventif sur le marché ne garantissait pas l'acquisition des médicaments, la prise de manière correcte de la dose par ces femmes, et la qualité des médicaments. Ainsi, dans certaines formations sanitaires, les femmes enceintes n'avaient plus bénéficié de traitement préventif intermittent depuis le début de la rupture de stocks.

Certains responsables de SSD semblaient justifier la distribution de routine de MILD et de traitement préventif intermittent pour les femmes enceintes par des critères autres que ceux concernant la lutte contre le paludisme.

Le problème est que la distribution de routine a été suspendue suivant un ordre «d'en haut» sous prétexte que la population a reçu trop de choses durant les campagnes et que ces moustiquaires offertes risquent d'être négligées par la population. (médecin)

De toutes les façons, peu importe que les femmes enceintes reçoivent ou non le kit préventif contre le paludisme!! Elles bénéficient déjà d'autres offres. Il n'y a pas que les moustiquaires et le traitement préventif intermittent! Premièrement, elles bénéficient toujours de toutes sortes de sensibilisation. Deuxièmement, si elles consultent dans un centre de dépistage $\mathrm{du} \mathrm{VIH} / \mathrm{sida}$ ',elles seront dépistées gratuitement. Elles y bénéficient aussi d'un dépistage de syphilis. La sulfadoxine-pyriméthamine ou les moustiquaires sont des bonus, s'il y en a de disponibles, sinon, rien. (cadre d'unSSD)

Tous les soignants étaient unanimes quant à lanécessité d'organiser des séances d'IEC. Mais, ils ont déclaré ne pas avoir le temps de se déplacer dans les fokontany pour le faire. De plus, les habitants ne leur semblaient pas vouloir consacrer du temps pour ce genre de discours. En revanche, certains soignants prenaient parfois le temps de diffuser les messages-clés autour du paludisme lors des consultations. D'autres intervenaient pour une brève IEC lors des rares événements sociaux qui réunissaient des gens (à la fête de l'école du village, par exemple).

Durant les 12 mois précédant l'enquête, la population d'Ankazobe avait étéplus souvent touchée par l'IEC (49,5\%) que celle de Brickaville (33\%; OR=0,50; IC $95 \%: 0,34-0,73$; $\mathrm{p}=0,002$; Figure 4). Dans l'ensemble, moins de la moitié de la population avait été touchée par au moins un message d'IEC durant les 12 derniers mois précédant l'enquête. Les médias d'IEC les plus cités étaient «les émissions radio » et «les affichages ». L'irrégularité de la couverture en réseau radio de la zone de Brickaville expliquait la faible exposition à l'IEC par rapport à Ankazobe. Cette faible couverture en éducation de la santé expliquait en partie la faible connaissance sur le paludisme par la population. Selon une autre étude, des habitants ont compris à travers la sensibilisation 
à la radio que le paludisme ne concernait que la capitale (Mattern et al., 2016), ce qui était faux.

Le graphique suivant récapitule le taux de couverture des actions préventives réalisées (taux d'utilisation des moustiquaires: $\mathrm{N}_{\mathrm{A}}=2101, \mathrm{~N}_{\mathrm{B}}=1940$; proportion de grossesses avec traitement préventif intermittent: $\mathrm{N}_{\mathrm{A}}=43, \mathrm{~N}_{\mathrm{B}}=19$; taux d'exposition aux IEC : $N_{A}=1066, N_{B}=1$ 107).

Figure 4. Taux d'utilisation et d'exposition aux mesures préventive

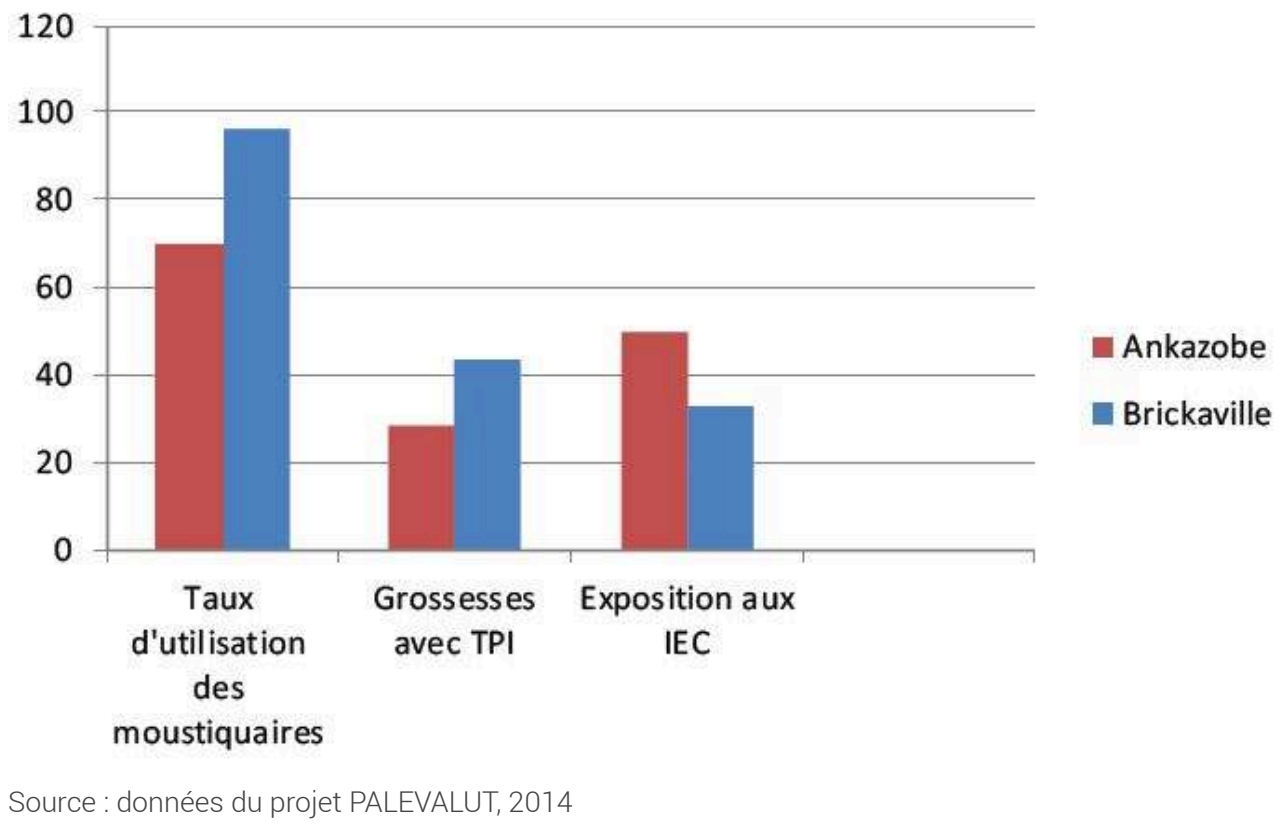

Parmi les mesures de lutte contre le paludisme, de la prise en charge des cas de fièvre aux mesures préventives, nombreuses étaient celles qui n'étaient pas mises en œuvre de façon appropriée ou en fréquence suffisante. Certaines étaient par ailleurs conçues d'une façon irréalisable sur le terrain par une population particulièrement démunie. Ces défaillances de l'organisation et de l'offre de soins et de services contribuaient à la vulnérabilité liée au paludisme de la population.

\section{Vulnérabilité liée aux comportements thérapeutiques et préventifs de la population}

Le comportement de recours aux soinsmédicaux dépendait de la représentation de la maladie et de l'accessibilité financière et physique de ces soins. Les modalités d'utilisation des MILD ne contribuaient pas souvent à la protection contre les piqûres de moustiques.

\section{1) Le recours aux soins}

L'analyse de recours aux soins portait sur les individus âgés de plus de 15 ans, ayant eu de la fièvre durant les trois mois précédant l'enquête (Figure 5). Globalement, l'automédication et le non-recours (ne pas se soigner) marquaient l'itinéraire thérapeutique de la majorité de la population. Le faible taux de recours aux 
tradipraticiens est présumé être lié à une sous-déclaration que l'approche qualitative nous a permis de comprendre. La consultation de tradipraticiens en cas de fièvre ou de paludisme a été mentionnée aussi bien par les soignants que par les autres répondants dans les entretiens. Le recours aux soins médicaux (y compris aux AC) n'étaitque de $35 \%$ à Ankazobe et de $60 \%$ à Brickaville. Le recours aux centres hospitaliers des districts concernait surtout les malades référés par les médecins des centres de santé de base pour complication du paludisme.

Figure 5. Fréquences des types de recours en cas de fièvre $\left(N_{A}=242, N_{B}=445\right)$

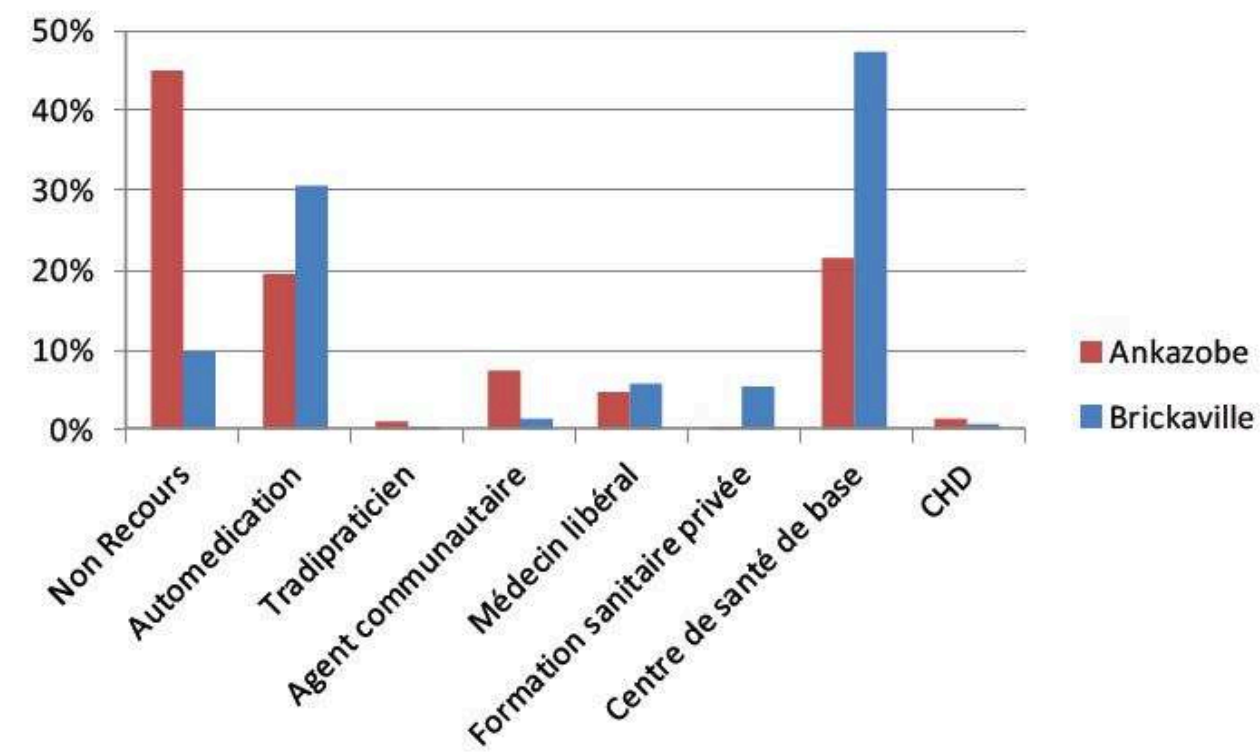

\section{2) La représentation du paludisme et la perception de la gravité de la fièvre}

Le choix du recours dépendait aussi de la représentation de la maladie et de la perception de la gravité des symptômes. Dans notre étude, la fièvre était perçue comme un état de santé anodin. La chaleur, l'exposition au soleil, le passage d'une saison à l'autre, la fatigue, étaient les causes les plus citées de la fièvre. Souvent, surtout chez les adultes, la fièvre n'était pas immédiatement traitée. Lorsqu'elle durait, elle était parfois traitée par du paracétamol pris en automédication. La fièvre prenait la qualification de tazomahery ("fièvre forte »)avec l'apparition d'autres signes de gravité et la persistance de la fièvre. C'est à ce stade que des noms de maladies étaient évoqués comme étant à l'origine du tazomahery, entre autres le tazomoka (signifiant " fièvre de moustique ») ou paludisme. Mais la majorité ne faisait pas le lien avec les moustiques malgré le nom de la maladie qui mentionne ce vecteur. La croyance au sortilège, à une malédiction à la suite d'infraction contre la morale ou le culte des ancêtres, conduisait au recours à des tradipraticiens. Par ailleurs, la convulsion était souvent perçue comme de la compétence des tradipraticiens. La dangerosité du paludisme était perçue par la connaissance de cas de décès du paludisme. La perception de la dangerosité du paludisme 
était associée positivement au recours aux professionnels de santé et était plus fréquente à Brickaville. De leur côté, les soignants se plaignaient du recours aux soins médicaux tardif des patients. Selon eux, les malades se présentaient souvent à un stade avancé de la maladie.

\section{3) Le coût du recours aux soins médicaux}

Le coût du recours était significativement plus élevé à Brickaville qu'à Ankazobe. Les soins médicaux professionnels étaient significativement plus chers que les autres types de recours. Cela suggère que le coût pouvait être l'un des principaux déterminants des recours (Figure 6).

Figure 6. Proportion des malades selon le coût (en Ariary ${ }^{8}$ ) et le type de recours aux soins, en cas de fièvre $\left(N_{B}=377, N_{A}=127\right)$

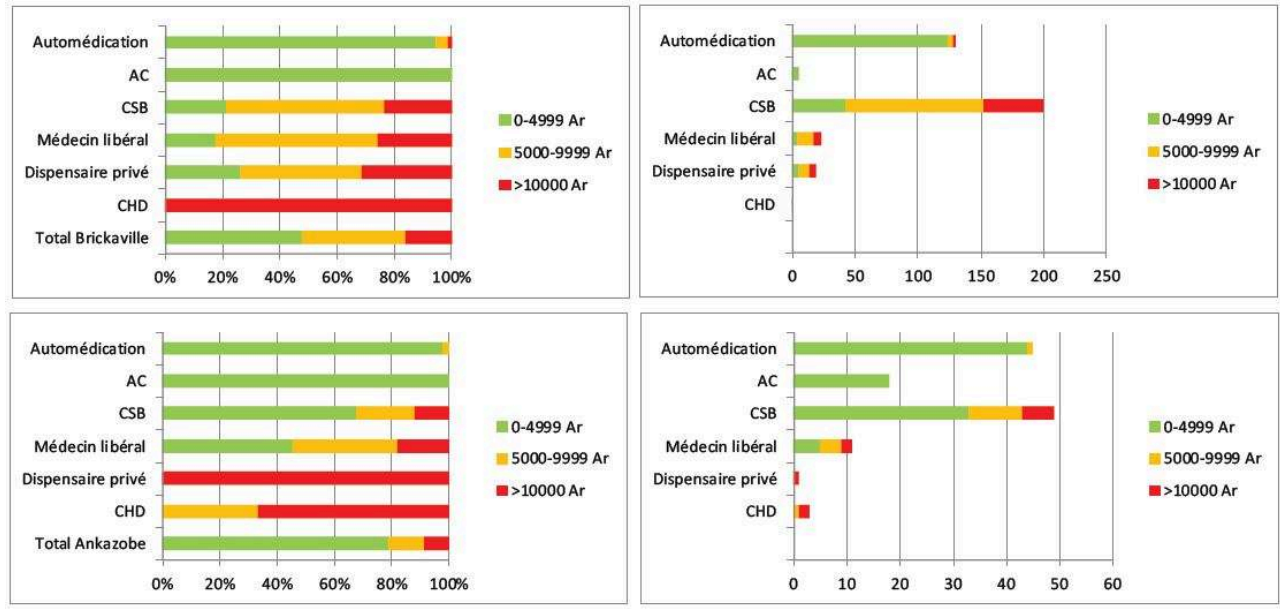

Source : données du projet PALEVALUT, 2014

49 Le coût de traitement du paludisme grave n'est pas pris en charge (ministère de la Santé publique de Madagascar, 2012). Ce coût incluait le prix des médicaments (autres que les ACT), les frais liés à l'hospitalisation, les autres frais indirects, particulièrement ceux de l'évacuation sanitaire en voiture vers un centre hospitalier.

Au moins, ils doivent dépenser dans les $200000 \mathrm{Ar}$ pour emmener le malade à Ankazobe. Savez-vous que la location de taxi-brousse de [cette formation sanitaire] à [l'hôpital de référence] coûte $150000 \mathrm{Ar}$ ? Le taxi-brousse doit être payé au nombre de places de la voiture. Une fois là-bas, ils doivent encore payer les soins, les médicaments et se débrouiller pour tout le reste. (médecin)

Le problème est que la famille doit prendre à sa charge le coût de l'évacuation. En cas de haute complication de la maladie, le malade doit être sous perfusion d'ici. La famille doit absolument louer une voiture pour l'emmener. Mais la location de voiture coûte entre 60000 et 100000 Ar. (médecin)

Ce coût de la prise en charge du paludisme grave contribue à la vulnérabilité liée au paludisme dans la mesure où il peut retarder ou ne pas permettre les soins dans les centres hospitaliers, et contraint les médecins communautaires à «bricoler » des soins et une prise en charge sur place, aux risques et périls des malades. 


\section{4) L’accessibilité physique des formations sanitaires}

51 Un autre déterminant majeur connu du recours aux soins est la distance à parcourir pour rejoindre un centre de santé (Dunn et al., 2006). Dans notre étude, cette distance a été mesurée en termes de durée de déplacement pour un trajet (Figure 7). Généralement, les habitants se déplaçaient à pied ou en pirogue de leur domicile au centre de santé de base. Le contexte financier de la population et l'enclavement ne permettaient pas des déplacements en voiture. La durée du trajet vers les centres hospitaliers (qui correspondent à la structure de santé la plus éloignée car se trouvant en ville), était généralement plus courte car les habitants ne s'y rendaient que pour une évacuation sanitaire, en voiture de location.

Figure 7. Proportion des malades selon la durée (en heure) d'un trajet et le type de recours pour fièvre, en cas de recours unique $\left(N_{B}=371, N_{A}=115\right)$

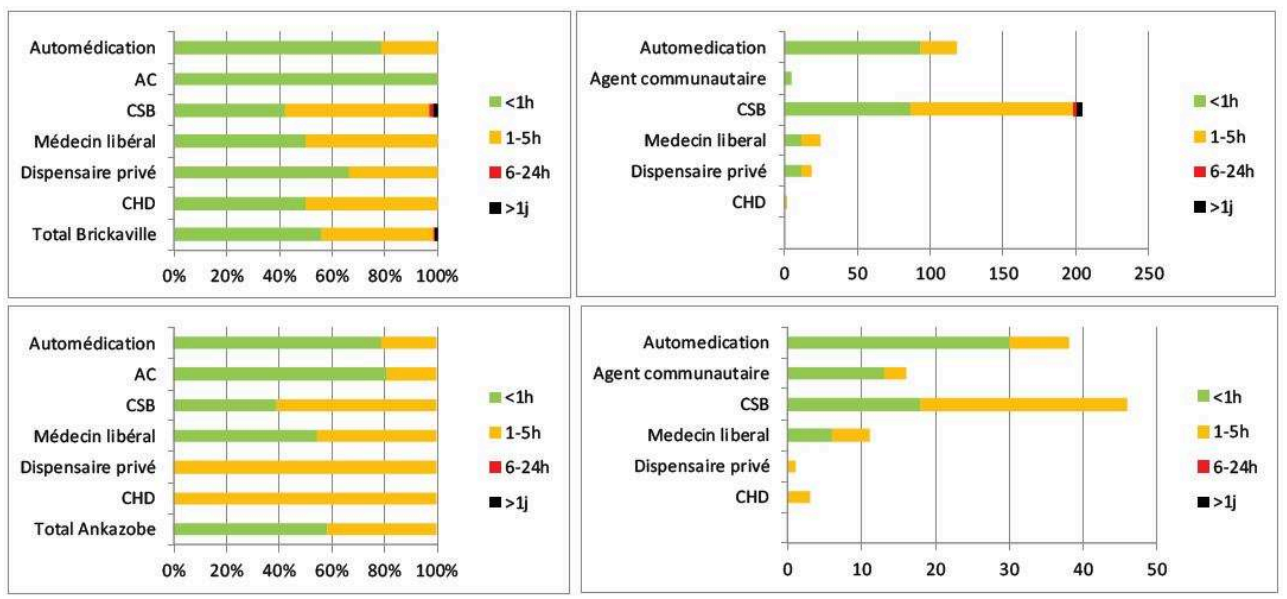

Source : données du projet PALEVALUT, 2014

52 La disponibilité desmédicaments, leur accessibilité sur le marché informel (dans les petites boutiques des villages et auprès des colporteurs) et la modicité de leurs prix favorisaient l'automédication.

Dans cette analyse des coûts et de distance, le faible recours aux AC était paradoxal ( $<8 \%$ des cas de fièvre ; Figures $5,6,7$ ). Rappelons que l'objectif de la participation des $\mathrm{AC}$ à la lutte contre le paludisme était l'amélioration de l'accès de la population aux soins médicaux. Ces AC sont des habitants présents au nombre de 3 à 5 dans chaque fokontany et leurs prestations sont théoriquement gratuites. Cependant, les AC faisaient souvent payer ${ }^{9}$ les ACT au prix de 100 Ar les doses de trois jours, accompagnés d'une plaquette de Paracétamol vendue à $50 \mathrm{Ar}$ le comprimé. Ces frais modiques ne constituaient pas un frein à la consultation, ils étaient similaires aux coûts de l'automédication, d'autant plus que les AC accordaient quasiment toujours un règlement à crédit.

En revanche, la limitation de l'offre de soins des AC aux enfants posait problème. D'ailleurs, les habitants et les AC auraient voulu que les AC puissent prendre en charge aussi les adultes. Dans la pratique, certains AC prescrivaient des médicaments pour les adultes, malgré la limitation de fonction et leur formation élémentaire.

Il y a beaucoup de cas de paludisme. Des adultes viennent me voir [...]. Ils se plaignent d'avoir des tremblements. Je leur fais un TDR. Puis je les envoie à l'hôpital 
avec la cassette [les résultats] pour qu'ils n'aient plus à faire un TDR une fois là-bas.

C'est tout ce que je fais mais je ne donne pas de médicaments. (AC)

Des fois, je leur [à des adultes] vends du Paracétamol. Je leur explique que j'ai peur

d'être sanctionnée si je leur prescris quelque chose, mais ils insistent... (AC)

Étant donné que la population connaissait les facilités financières offertes par les AC, leur capacité à utiliser des TDR ou à prescrire des ACT, et la disponibilité des médicaments à coûts modestes chez ces agents, notre analyse présume que la principale explication du faible recours de la population aux AC en cas de fièvre était la limitation de l'activité officielle de ces derniers à la prise en charge des enfants de moins de 5 ans, alors que le paludisme touchait largement aussi les individus des autres classes d'âge.

Peut-être existe-t-il aussi des raisons psychologiques, éventuellement en relation avec la confiance que les AC inspirent à la population pour la soigner ? En effet, dans notre étude, les enfants, présumés plus fragiles, étaient plus souvent conduits dans les centres de santé que les adultes. Cela pourrait être lié à la représentation des médecins comme offrant un meilleur service et une meilleure sécurité que les AC (Pourette, Mattern et Raboanary, 2013; Rogier et al., 2013). La question n'a pas pu être approfondie dans cette étude.

\section{5) Mode d'utilisation des MILD}

57 Le taux d'utilisation des moustiquaires (Figure 4) ne peut suffire pour évaluer le niveau de protection contre les piqûres de moustiques. Pour ceux qui utilisaient les MILD, les modalités d'utilisation de ce dispositif ont été analysées. L'heure habituelle approximative à laquelle les individus commençaient à entrer effectivement sous les moustiquaires, ainsi que l'heure habituelle approximative à laquelle ils les quittaient au matin, ont été demandées. Tenant compte des heures auxquelles les anophèles piquaient le plus (Lundwall et al., 2005), nous avons présumé qu'être sous moustiquaires entre 20 heures au plus tard le soir et 6 heures au plus tôt le matin, et ce « tous les jours de l'année ", protégerait des piqûres des vecteurs du paludisme.

Bien que les enfants de moins de 5 ans fussent relativement mieux protégés, le niveau de protection contre les piqûres de moustiques était faible. Le niveau d'exposition aux piqûres de moustiques augmentait avec l'âge. Les entretiens ont révélé que les enfants et les femmes enceintes étaient prioritaires pour utiliser les moustiquaires lorsqu'elles étaienten nombre insuffisant dans le ménage. (Tableau 1)

Tableau 1. Proportion d'individus présumés protégés des piqûres de moustiques (en \%)

\begin{tabular}{|l|l|l|l|}
\hline & N & \% & IC 95 \% \\
\hline Total & 3553 & 33,2 & $27,3-39,6$ \\
\hline Ankazobe & 1672 & 26,6 & $20,8-33,4$ \\
\hline Brickaville & 1881 & 39 & $29,7-49,2$ \\
\hline Âge & & & \\
\hline
\end{tabular}




\begin{tabular}{|l|l|l|l|}
\hline$<2$ ans & 198 & 62,6 & $51,9-72,2$ \\
\hline $2-4$ ans & 438 & 58,9 & $50,5-66,8$ \\
\hline $5-9$ ans & 562 & 47,9 & $40,4-55,4$ \\
\hline $10-14$ ans & 458 & 33,2 & $23,4-44,7$ \\
\hline $15-19$ ans & 299 & 23,4 & $15,6-33,6$ \\
\hline $20-49$ ans & 1235 & 18,1 & $13,6-23,8$ \\
\hline 50 ans + & 363 & 22,3 & $15,7-30,8$ \\
\hline
\end{tabular}
l'automédication, le non-recours aux soins de professionnels de santé ou d'AC, le recours tardif aux centres de santé (Mattern et al., 2016) sont des comportements qui pourraient être corrigés par une meilleure adaptation des messages d'IEC et un renforcement de leur fréquence.

\section{Conclusion}

62 L'approche mixte a permis de montrer qu'à Ankazobe et à Brickaville, la vulnérabilité liée au paludisme persistait malgré les efforts de lutte consentis. Les conditions écologiques et bioclimatiques de la transmission du paludisme sont invariantes mais n'ont été que partiellement contrebalancées par les activités de lutte. Cette étude a permis d'identifier quelques pistes d'amélioration de cette lutte: étendre la population-cible des AC au-delà des enfants de moins de 5 ans, moyennant une formation ajustée, permettrait d'augmenter l'impact des interventions; envisager la prise en charge du paludisme grave au niveau des centres de santé de base, moyennant la formation et l'équipement des soignants, permettrait de prendre en compte les contraintes financières de la population, leurs difficultés de déplacement ainsi que les contraintes logistiques des médecins communautaires; identifier les failles dans la planification et la mise en œuvre des actions de lutte (pour éviter les ruptures de stocks d'intrants) permettrait de mieux assurer leur efficacité. Cette étude indépendante des institutions en charge de la lutte antipaludique permet

Populations vulnérables, 5 | 2019 
d'en identifier les forces et les faiblesses et de proposer des solutions adaptées au contexte épidémiologique et socioculturel local.

\section{BIBLIOGRAPHIE}

AIMS (2011), « L'utilisation des méthodes mixtes dans la recherche française en stratégie : constats et pistes d'amélioration ", XX Conférence de l'Association Internationale de Management Stratégique, Nantes, AIMS, p. 1-26.

Angers M. (1996), Initiation pratique à la méthodologie des sciences humaines, Anjou (Québec), Éditions CEC.

Aubry P. et Gaüzère B.-A. (2015), « Paludisme Actualités 2015 », Médecine Tropicale, p. 1-25.

Becerra S. (2012), « Vulnérabilité, risques et environnement : l'itinéraire chaotique d'un paradigme sociologique contemporain », Vertigo - la revue électronique en sciences de l'environnement, vol. 12, $\mathrm{n}^{\circ} 1$.

Bileckot R., Mbouolo T., Ntsiba H., Fouty-soungou P. et Fila A. (1992), « Facteurs de paralysies sciatiques secondaires aux injections intra-musculaires ", Médecine d'Afrique Noire, vol. 39, n² 2, p. $129-132$.

Direction de la veille sanitaire et de la surveillance épidémiologique (2012), «Évolution du nombre des cas du paludisme à Madagascar (2000 à 2011) ", in $3^{e}$ Journée de la Société de Pathologie Infectieuse de Madagascar, 2012, Antananarivo.

Dunn J. R., Frohlich K. L., Ross N., Curtis L. J. et Sanmartin c. (2006), « Role of geography in inequalities in health and human development ", in Heymann J. et al. (dir.), Healthier Societies: From Analysis to Action, Oxford, Oxford University Press, p. 237-266.

GF-OIG-13-052 (2014), « Procurement activities of the principal recipients of the Global Fund's national strategy application grant for malaria to Madagascar. Investigation report ».

Houéto D., D'Hoore W. et Deccache A. (2008), « Perceptions de la participation des parents par les professionnels de santé à la lutte contre le paludisme de l'enfant au Bénin ", Santé Publique, vol. $20, \mathrm{n}^{\circ} 1, \mathrm{p} .19-28$.

Hunt E. et Lavoie A.-M. (2011), « Les méthodes de recherche quantitatives et qualitatives peuvent-elles déjà coexister ?", Recherche en soins infirmiers, $n^{\circ}$ 105, vol. 2, p. 25-30.

INSTAT (2010), « Enquête démographique et de santé Madagascar 2008-2009 ».

Lundwall E., Pennetier C., Corbel V., Ludovic De Gentile et Legros F. (2005), « Paludisme : se protéger des piqûres d'anophèles ", La revue du praticien, $n^{\circ}$ 55, p. 841-848.

Mattern C., Pourette D., Raboanary E., Kesteman T., Piola P., Randrianarivelojosia M. et Rogier C. (2016), « "Tazomoka is not a problem”. Local perspectives on malaria and impact on fever case management and bed net use in Madagascar », PLoS ONE 11(3): e0151068. https:// doi.org/10.1371/journal.pone.0151068, p. 1-14. 
Ministère de la Santé publique de Madagascar (2012) «Plan stratégique de lutte contre le paludisme Madagascar 2013-2017. Première version ».

Ministère de la Santé publique de Madagascar, roll back malaria (2016), «Plan de communication pour la lutte contre le paludisme 2013-2017. Deuxième version ».

OMS, (2015), « Stratégie technique mondiale de lutte contre le paludisme 2016-2030».

Pluye P. (2012), « Les méthodes mixtes pour l'évaluation des programmes », in Ridde V. et Dagenais C. (dir.), Approches et pratiques en évaluation de programme, Montréal, Presses de l'université de Montréal.

Pluye P. et Hong Q. N. (2014), « Combining the power of stories and the power of numbers: mixed methods research and mixed studies reviews ", Annual Review of Public Health, vol. 35, p. 29-45.

Pourette D., Mattern C. et Raboanary E. (2013), « Rapport de mission de l'étude qualitative (Medali)».

Rakotomalala R. S., Randriamihangy N., Zara A. N., Andrianarivelo A., Alson O. R. et Rasamindrakotroka A. (2015), «Paludisme observé chez les patients fébriles au Centre de santé materno-infantile de Moramanga en 2007-2009 », La Revue médicale de Madagascar, vol. 5, n 1, p. 516-519.

Ridde V. (2013), «Personnes vivant avec le VIH, méthodes qualitatives et couvertures universelle en santé ", Sciences Sociales et Santé, vol. 31, n³, p. 29-37.

Rogier C., Randrianarivelojosia M., Kesteman T. et Vigan I. (2013), « Document de travail : Résultats des études épidémiologiques du projet MEDALI ».

\section{NOTES}

1. L'objectif du projet PALEVALUT était de mettre au point, valider et publier, une méthodologie pluridisciplinaire intégrée («boîte à outils ») permettant l'évaluation de l'efficacité en conditions réelles des stratégies de lutte contre le paludisme et l'identification des facteurs interférant avec le déploiement et l'efficacité de ces stratégies. http://www.palevalut.org. L'étude PALEVALUTMadagascar a été réalisée au sein de l'Unité de paludisme et de l'Unité d'épidémiologie de l'Institut Pasteur de Madagascar.

2. Les centres de santé de base (CSB) sont les formations sanitaires existantes au niveau des communes.

3. Les agents communautaires (AC) sont des habitants choisis par les responsables des centres de santé de base. Ils se voient dispensés une formation élémentaire pour la prise en charge des maladies de l'enfant de moins de 5 ans. En matière de paludisme, les AC sont habilités à faire un TDR, à prescrire des ACT en cas de paludisme simple et du paracétamol pour faire baisser la température. Les cas de paludisme grave sont à signaler dans les centres de santé.

4. À Madagascar, le fokontany est la dernière subdivision administrative et géographique.

5. D'après les données quantitatives de PALEVALUT sur la disponibilité d'intrants, trois formations sanitaires sur quatre ont connu des ruptures de stocks d'intrants. La durée de rupture pouvait dépasser un an.

6. Le traitement du paludisme par injection de quinine est discuté supra.

7. Les formations sanitaires investiguées ne faisaient pas partie des centres de dépistage du VIH/ Sida.

8. Ariary (Ar) est la monnaie malgache. Le revenu moyen par habitant était proche de $2518 \mathrm{Ar} /$ jour, en 2012. En 2014, 1 euro =3 $200 \mathrm{Ar}$. 
9. Les AC constituaient des stocks de médicaments chez eux par leurs propres fonds. Ils se faisaient rembourser en vendant aux prix d'achat aux personnes qui consultaient chez eux. Lorsque les ACT et les TDR utilisés par les AC étaient fournis par les centres de santé (quand les stocks le permettaient), les prix modiques étaient toujours appliqués et constituaient un maigre profit pour les AC. Ce gain était approuvé par les responsables des SSD, compte tenu du statut de bénévole des AC.

\section{RÉSUMÉS}

Le risque de décès, d'aggravation d'une maladie ou d'endettement dans ses suites, dépend des dispositifs et moyens disponibles et accessibles à la population pour y faire face. Ainsi, la vulnérabilité liée au paludisme dépend des contextes socio-économique et géoclimatique qui déterminent le risque de transmission, des comportements individuels ou collectifs, et des moyens des ménages pour faire face à la maladie, la prendre en charge ou la prévenir.

La présente étude vise à identifier les déterminants de la vulnérabilité liée au paludisme de la population de Madagascar, en analysant le contexte et les comportements de prévention et de recours aux soins médicaux en cas de fièvre. Une étude quantitative sur 4043 personnes de 803 ménages a été combinée à une étude qualitative reposant sur une soixantaine d'entretiens, dans deux districts, Ankazobe (Hautes Terres) et Brickaville (côte est), où le risque de paludisme et les contextes psycho-sociaux différaient. Les données ont été collectées en 2014 dans le cadre d'une thèse et d'une étude pluridisciplinaire, multicentrique et internationale (projet PALEVALUT ${ }^{1}$ ), pour laquelle un questionnaire et des guides d'entretien ont été développés, standardisés et validés.

À Brickaville, les conditions bioclimatiques (chaudes et humides) sont plus favorables au développement des moustiques vecteurs du paludisme qu'à Ankazobe. La survenue de fièvre, la connaissance d'une personne décédée du paludisme, la perception de la dangerosité de cette maladie, la citation de fausses croyances à son sujet, l'utilisation de moustiquaires, le recours à un professionnel de santé et à un test sanguin pour son diagnostic, et le paiement de frais pour le déplacement vers une structure de santé ou pour obtenir des soins en cas de fièvre, y étaient significativement plus fréquents. En revanche, l'exposition à l'éducation pour la santé, la compréhension du paludisme, le recours aux agents communautaires et la prise d'une combinaison thérapeutique à base d'artémisinine en cas de prise d'antipaludique, y étaient significativement moins fréquents qu'à Ankazobe.

Ainsi, à Brickaville où la prévalence du paludisme était la plus élevée, certaines connaissances, attitudes et pratiques, associées à la prévention ou au traitement étaient plus fréquentes alors que d'autres, notamment en matière thérapeutique, étaient plus souvent inappropriées. L'écosystème, la disponibilité, l'accessibilité et la nature des lieux de recours, l'exposition à l'éducation pour la santé, les pratiques médicales des soignants et des patients, différaient significativement dans ces sites distants de moins de $300 \mathrm{~km}$ ou entre des sous-populations d'un même site. Ces déterminants de la vulnérabilité liée au paludisme, multidimensionnels et complexes, sont présentés et discutés ici sur les plans quantitatifs et qualitatifs. 
INDEX

Mots-clés : paludisme, fièvre, vulnérabilité liée au paludisme, recours aux soins, prévention, Madagascar

Keywords : malaria, fever, vulnerability linked to malaria, seeking treatment, prevention, Madagascar

\section{AUTEURS}

\section{ANDRY HERISOA ANDRIANASOLO}

LIR3S-UMR 7366, Institut Pasteur de Madagascar

\section{EMMA RABOANARY}

Institut Pasteur de Madagascar

\section{CHIARELLA MATTERN}

Institut Pasteur de Madagascar

THOMAS KESTEMAN

Institut Pasteur de Madagascar

\section{DOLORÈS POURETTE}

IRD - CEPED UMR 196

\section{CHRISTOPHE ROGIER}

Service de santé des Armées, ministère de la Défense 